\title{
Designing and Doing Laboratory Experiments ${ }^{1}$
}

Tobias Wolbring, Chair of Empirical Economic Sociology, FAU Erlangen-Nürnberg

\section{Introduction}

In the previous chapters we have introduced the general methodological background for designing and doing experiments. Based on this, we illustrate in the following chapters how to conduct experiments in different settings and highlight potential pitfalls as well as important ethical and practical aspects of each type of experiment. In this chapter, we start with the most well-known type of experiment: the laboratory experiment. Laboratory experiments contain the three design elements common to all experiments - manipulation, group comparison, and randomization- and are staged in the rather sterile, anonymous, and controlled setting of a laboratory. As a laboratory (in the following lab), we understand a room that is specifically designed for conducting experiments in order to vary treatments of interest while controlling for external nuisances. The lab environment thus provides not only the advantage of good replicability, but gives researchers strict control over setting, treatment manipulation, and measurement of independent and dependent variables.

Due to this high degree of replicability and control, the lab is often perceived as the prototypical location for conducting experiments. Although other types of experiments are nowadays frequently used in the social and behavioural sciences), many researchers automatically think about lab experiments when hearing the term "experiment". Criticism questioning the contribution of experiments to social science research questions is hence often actually directed towards lab experiments and not towards experiments in general. At the same time, imagining the ideal conditions of a controlled lab helps to see deviations of experiments in other settings from this ideal more clearly. Hence, irrespective of whether one actually plans to conduct an experiment in the lab or in a different setting, firm knowledge of lab experiments is essential to avoid pitfalls and to anticipate potentially scepticism.

Let us start by illustrating the lab experimental approach with the following example: In a famous experiment on interpersonal influence and group pressure, the social psychologist Solomon Asch (1951) invited male college students to a lab and asked them to compare a visually displayed line with three comparison lines. The task was to identify among the three comparison lines the one matching the displayed line. As illustrated in Figure 1, there was not

\footnotetext{
${ }^{1}$ This is a preliminary draft of a chapter originally planned for a textbook on "Designing and Doing Experiments". I am grateful to Alysha Owen and Stefanie Eifler for helpful input and hope that the text turns out useful for anyone who is planning to conduct an experiment or who is teaching a class on experimental methods.
} 
much ambiguity involved in this simple task. As a result, subjects solved the task on their own with an error rate close to zero (95\% of subjects gave no wrong answer in any trial). These judgements in absence of others served as the control condition in Asch's experiment providing a benchmark for the experimental condition.

Figure 1: Line Judgement Task in the Asch Experiment
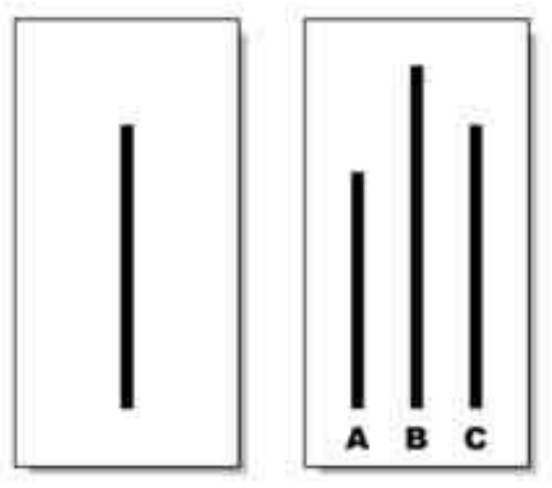

Source: McLeod (2008)

In the experimental condition, subjects sat next to seven other persons in the lab - all of them part of the research team, but framed as additional subjects. One after the other, these confederates of the experimenter announced their judgement aloud. As instructed by the experimenter, all confederates gave the same wrong answer on purpose in some of the trials with the actual subject being last in line. This unanimous majority judgement created group pressure for the subject and served as the treatment in the experiment (with further variations regarding group size and degree of uniformity). Under group pressure only $26 \%$ of the subjects gave correct answers in all trials with individual judgements moving in the direction of the majority. Comparing this outcome with the control condition (26\% vs. 95\%) underlines the strong effect of social pressure on individual judgements. Asch's simple, but path-breaking laboratory experiment thus shows how a rather abstract and artificial task in the lab helps to gain insights into human behaviour (for a meta-analysis of replications and extensions of the original experiment see Bond and Smith 1996). At the same time, the study design raises issues which are rather common in lab experimental research such as questions about ethics, deception, and the generalizability of findings beyond student samples.

In this chapter, we explain in more detail how to conduct experiments in the laboratory and how to address these issues. We frame the lab experiment as a social situation consisting of 
interactions before, during, and after the experiment and give recommendations on how to handle the different stages. Following the programmatic contribution by Falk and Heckman (2009: 535), we thereby argue, that "lab experiments are a major source of knowledge in the Social Sciences", even though they are - as every method - no silver bullet (see also Bless and Burger 2016). We thus conclude the chapter by highlighting pitfalls regarding treatment, group comparisons, and randomization and discuss ethical and practical challenges of conducting experiments in the lab.

\section{The lab experiment as a social process}

To learn how to conduct lab experiments, it is helpful to distinguish different stages of designing and doing experiments in the lab. In this section, we will discuss each of the following four steps depicted in Figure 2 in more detail: (a) creation of a social system in the lab and design of treatments, (b) recruitment of subjects, (c) actual experiment in the lab, and (d) debriefing and payment of participants. In our presentation, we will put a particular emphasis on the social processes involved, especially the social interactions between experimenters and subjects before, during and after experiments in the lab.

Figure 2 Stages of lab experiments

$\begin{aligned} & \text { (a) Create an artifical social system, } \\ & \text { think about interventions and } \\ & \text { decide how to measure outcomes }\end{aligned}$
(b) Recruit subjects, assign them to
treatment conditions and invite
them to participate


(a) A first step in planning an experiment is to think about how to create an artificial social setting in the lab that mirrors a real-world situation of interest. Answering research questions in the Social Sciences by means of lab experiments requires to create an "idealized social system" (Willer and Walker 2007), to intervene in this system by changing the value of core explanatory factors - such as institutions, network structures, or distribution of resources - and then to measure the outcome variable of interest. Lab experiments therefore always abstract from the complexities and dynamics of social reality to a certain degree to exploit the rather stable and controlled setting of the lab. Although sometimes perceived as a weakness of lab experiments, artificiality, sterility, and tight control over the setting are actually major strengths of lab experiments because they offer advantages regarding manipulation of independent variables, measurement of outcomes, and isolation of treatment effects. At the same time, the idealized social system should contain essential components of the social world in order to provide insights generalizable beyond the abstract, idealized, and artificial lab situation.

The lab experiments of Wolbring and Riordan (2016) provides an example of what abstraction from the social world and creation of an idealized social system means in practice. We were interested in how physical attractiveness of instructors influences students' evaluations of teaching: Do attractive instructors receive better ratings? Or are they perceived as less competent? In order to explore these causal questions, we invited students to the lab and showed them randomly assigned CVs of hypothetical instructors containing a photograph. As can be seen in Figure 3, the photographs in the CVs functioned as the experimentally varied treatments depicting males and females being more or less physically attractive. Students then listened to an audio record of a lecture of the assigned hypothetical instructors and evaluated the teaching. Although actual lectures or video lectures would have increased realism of the setting, the researchers instead decided to use CVs with photographs and audio records to secure maximum comparability across experimental conditions and to avoid biases caused by hard to control variables such as instructors' gestures and voice. In line with previous observational research (Hamermesh and Parker 2005), the experiment showed that physical attractive instructors received significantly and markedly better ratings than less attractive ones. Because this finding applies even though all students listened to the same lecture, this example clearly demonstrates that creating an artificial decision situation in the lab can help to tease apart the effects of interest from other nuisances.

Figure 3 Example of a Hypothetical German CV 


\section{Lebenslauf}

Name: Dr. Alexander Kramer

Geburtstag: 25.06.1977

Geburtsort: Heidelberg
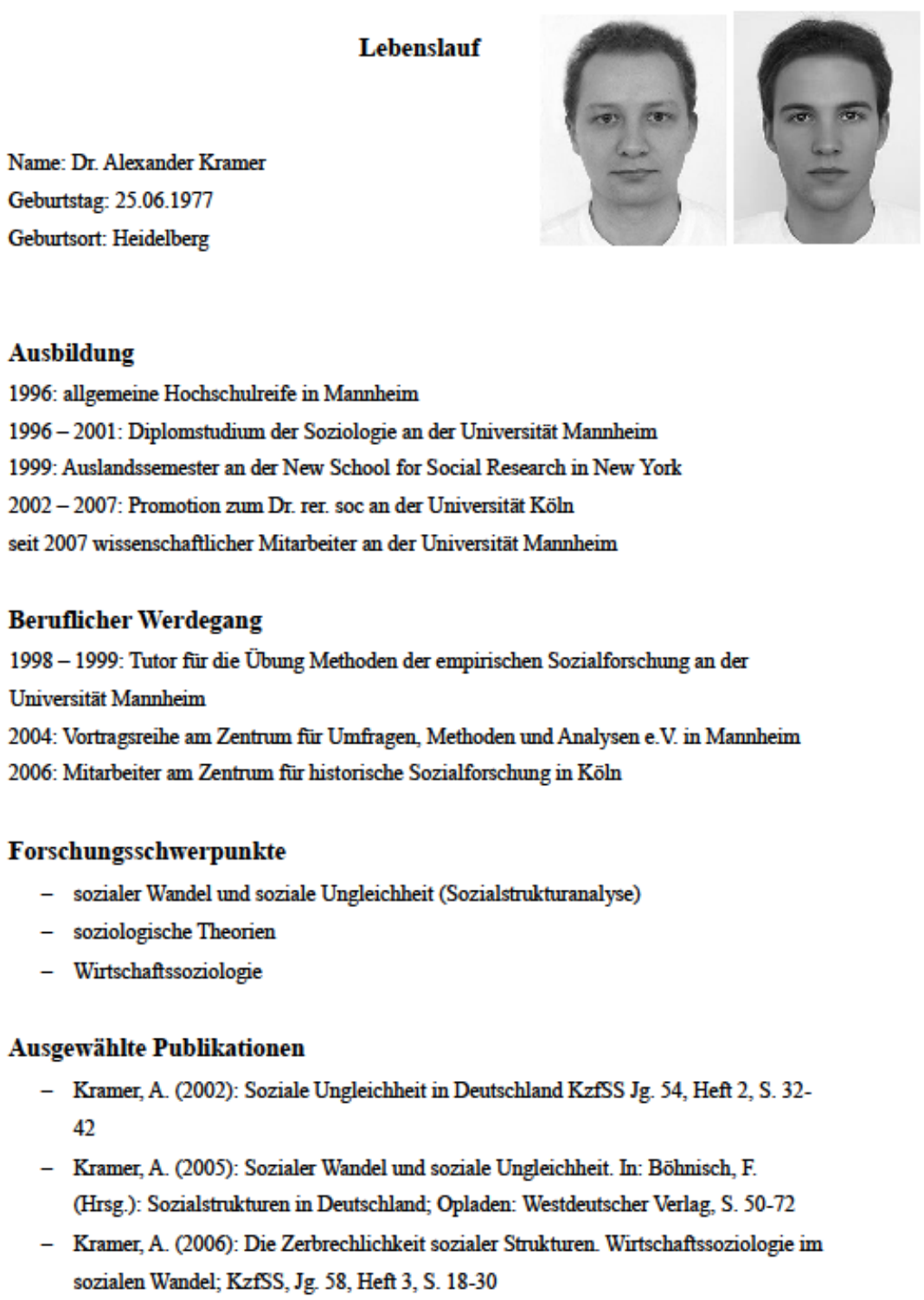

(b) In a second step, researchers need to recruit subjects for the experiment. In a world without restrictions, one would want to sample participants from the population of interest. In the above experiment on teaching evaluations, the population of interest were students, but usually it is the general population or some group of professionals such as bankers, criminals, or cab drivers. Such ideal sampling from the population of interest is rarely feasible in practice, because participation in lab experiments is time consuming for subjects and involves both direct (ticket for traveling to the lab) and opportunity costs (loss of time). As a consequence, it is much more common in practice to sample from student populations (for exceptions using non-student samples see Fréchette 2015). Many experimental labs in the social and behavioural sciences 
administer so-called subject pools consisting of persons - mostly current or former students who signalled their willingness to participate in experimental studies. Although these subject pools need to be refreshed from time to time due to attrition (e.g., email address changes, graduation, moving away), they are often a great starting point and indispensable resource for recruiting subjects. In addition, it is common practice in some research areas to require students in classes to participate in experiments to get hourly credit as a subject (for a discussion of this practice see section 4). Starting from an existing or newly created subject pool, one draws samples for the study and randomly assigns subjects to experimental conditions. Thereby, group sizes per experimental condition should be determined in advance based on a power analysis taking into account expected effect size and effect heterogeneity.

(c) Next, subjects are invited to participate in the study. At this point, it is necessary to provide information to the invited participants on some basic facts of the experiment such as aim of the study, duration of the experiment, and payment details. Taking into account that subjects' knowledge of the study topic might influence their willingness to participate and also their behaviour in the lab (see section 3), researchers might want to conceal the actual aim of the experiment at this stage of the study. For example, Asch (1951) didn't tell the participants of the aforementioned experiment (see section 1) that the study is about group pressure. In contrast he asked them to take part in a study on line judgement. Moreover, ethical considerations need to be taken into account when deciding about what to tell subjects upon invitation to the lab (for a more detailed discussion on ethics see section 4): If the experiment involves risks, subjects, of course, need to be informed about the potential downsides of participation in advance to make an informed choice.

Nowadays, potential subjects are usually invited via email and, when clicking on a link, are directed to a website where they can choose between different time slots for the experiment. If the random assignment to an experimental condition was already determined at the invitation stage, it is essential that the available choices offered to the subjects on the website are restricted to time slots for the assigned experimental condition. However, in some lab experiments it is also sufficient to randomly assign subjects to conditions upon arrival at the lab. Furthermore, it needs to be taken into account that usually only a small fraction of the invited subjects actually enrols in one of the experimental sessions. Thus, when setting up the subject pool one should be aware that the number of invited subjects needs to be much larger than the actual sample required for conducting the experiment. 
This also holds true for another reason: Even if subjects have accepted the invitation and have enrolled online for an experimental session, it cannot be taken for granted that they show up. Some arrive late or mix up the date, others don't come at all. In particular for experiments that require a fixed group size, such no-shows can cause serious problems. In such cases, besides having contingency plans for other group sizes, it can be a wise choice to invite more subjects to the session than actually needed. If too many subjects show up, some of them cannot participate in the session and need to be compensated for their efforts with a so-called show-up fee (for details see paragraph d). However, on average, this is still cheaper than sending subjects away because of insufficient group size.

(c) In a third step, the experimenter welcomes the subjects and seats them in the lab. It is highly recommended to researchers to have a clear idea about the sequence of the different steps of the experiment. As Shelly (2007) points out, the analogy between an experiment and a theatre performance provides helpful insights. Both call for a clear protocol on the dramaturgy of events, involve a "frontstage" and "backstage", and require a clear division of labour among the staff. In addition, experimenters need to be prepared to improvise and to have contingency plans - for example, when unexpected questions by the subjects come up, interruptions occur, participants don't adhere to the experimental protocol, bugs in the experimental software or other technical problems occur. To minimize these risks and to get a better idea about the procedure and length of the experiment, we recommend conducting several pilot runs before starting the actual experiment.

Researchers further need to think about the adequate physical setup in the laboratory, in particular how to secure a high degree of situational control while at the same time allowing for the social interactions of interest. The exact physical setup needs to be tailor-made to the specific research question and sometimes can be kept pretty simple or needs to be more complex. An example for the former is provided by the Asch experiment: Subjects entered a room with eight chairs and a projector and were seated next to each other. Because all participants except for one were confederates of the experimenter and followed a clear protocol, this simple setup allowed for a high degree of situational control minimizing nuisances and unwanted interactions.

However, experiments usually involve more than one subject at a time. Researchers thus need to carefully consider whether and in which way subjects are allowed to interact with each other. This is important to rule out unwanted social influences und to systematically structure interactions among subjects. Experimental setups in the lab can thereby range from open 
discussions over group problem solving making in a highly structured task to individual decision making.

Especially in the latter type of task, but also in various forms of group decision making it is essential to rule out unwanted social interactions. For this reason, many social scientific laboratories have detached workstations in order to minimize communication and coordination between subjects (for an exemplary lab see Figure 4). Besides these physical measures to avoid unwanted nuisances, in many social science experiments subjects are not allowed to directly interact with each other during the experiment (no communication rule). Instead they are often lead through the experiment on a computer screen and frequently only have computer-mediated interactions with each other. For example, in the ultimatum game developed by Güth et al. (1982) subjects are matched with anonymous strangers and can propose a division of a fixed amount of money (e.g. offering a 50/50 split of 10 Euros) among them entering the proposal in the computer. The other sees the offer on the screen and can either accept or reject the proposal with the latter choice leaving both persons without any gain.

Figure 4 Exemplary laboratory for Social Science experiments

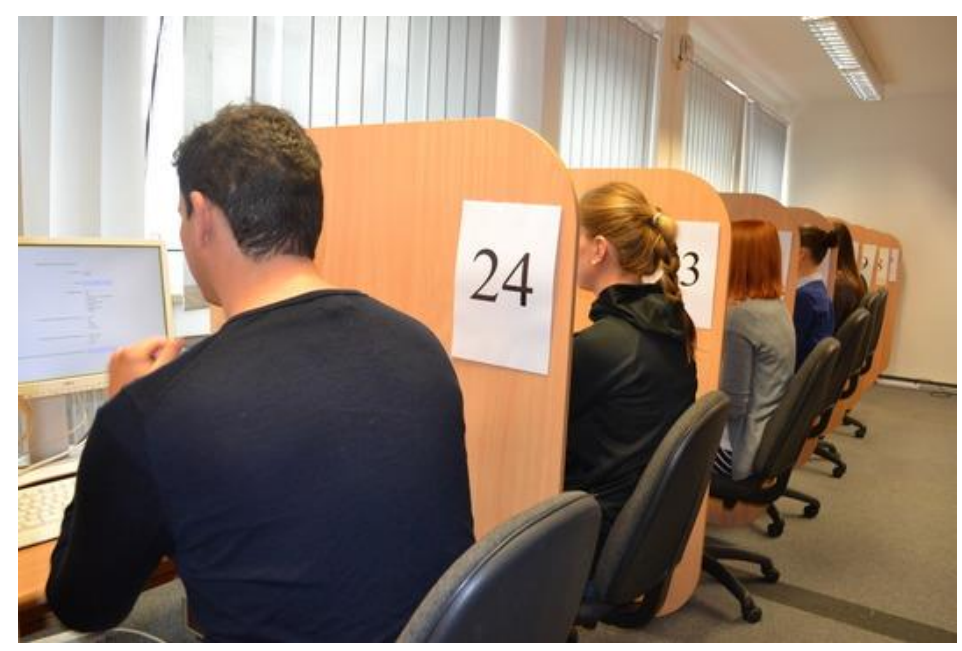

Source: https://www.melessa.uni-muenchen.de/profil/index.html

In the beginning of the session, the experimenter introduces the topic of the study, informs about the schedule, and explains how the experiment works, especially which tasks the subjects need to complete and of how many rounds the experiment consists. The introductory text and instructions should be as standardized as possible and may be even read from a sheet in order to rule out that subtle differences in wording convey moral value and influence participants' behaviour in the different experimental sessions. In addition, subjects usually also receive 
written instructions to increase comprehension and to clarify question which might arise during the course of the experiment. In case of complex experiments, it is highly recommended to walk the participants through hypothetical examples or to conduct test runs with them before starting the actual experiment to rule out misunderstandings. However, when choosing examples, one needs to be careful not to provide clues on "correct", socially desirable, or inadequate behaviour. For example, the experimenter might increase the salience of a "fair" 50/50 split in the aforementioned ultimatum game by using this split as an example. Moreover, subjects can learn in test runs about how to behave or how potential interaction partners behave. For example, subjects will abstain from offering a 90/10 split in the ultimatum game, if they learn in test runs that others always reject this offer.

(d) At the end of the experiment, subjects need to be debriefed about the actual aim of the study and its (expected or actual) results. Sometimes a direct debriefing after the session about the exact topic and related hypotheses motivating the experiment is problematic and can cause major problems, if the information spills over to future participants of the experiment. Such information diffusion is particularly likely if subjects know each other or if subjects of different sessions directly interact with each other because the sessions are scheduled immediately after each other. If spillover effects appear likely, we recommend to either take measures which reduce the likelihood that subjects from different experimental sessions interact with each other (e.g., by increasing time gaps or having separate doors for entering and existing the laboratory) or to delay the detailed debriefing to a later point in time (e.g. by sending a short report via email at the end of the whole experiment).

Moreover, for some lab experiments the study doesn't end for all subjects at the same time. Some subjects might complete the task or a questionnaire faster than others; sometimes some subjects need to complete additional tasks or are required to answer additional survey questions. Subjects leaving early or sitting in the lab without a task at hand can create unnecessary disturbances in the controlled lab setting. Thus, besides the "no communication" rule usually recommended in the lab, researchers might consider to provide additional tasks to entertain these subjects and to keep them from disturbing the other participants.

Finally, if monetary incentives are involved, ${ }^{2}$ subjects receive payment at the end of the experiment. Payment schemes should be determined on the basis of two considerations. First,

\footnotetext{
${ }^{2}$ Disciplines have different standards whether or not to pay subjects for participation in the experiment. In economics, the use of monetary incentives for participating in the experiment and performing well are obligatory. In psychology, payment is less frequently used and subjects often need to take part in experiments due course
} 
payment should compensate for direct and indirect costs of getting to the lab as well as time and effort for participating in the experiment (show up fee). Second, proper incentives should be given to take the experiment seriously and to have a sufficient motivation to perform well in the experimental task. The information how much a subject earned in the experiment should be treated strictly private and confidential, since some subjects will not want the other subjects to know how much money they made in the experiment. This is particularly true if earned payment allows inferences about individual choices in the experiment and if some types of behaviour are morally charged. Imagine, for example, an egoistic proposer in the ultimatum game who always offers a 90/10 split keeping most of the profit to himself. Hence, subjects should be called to the paying office separately and receive the earned money in a private setting. It is worth emphasizing that subjects should be informed about the payment procedure already in the beginning of the experiment, because the information that no one else will know how much they earned might make a difference in how they behave in the experiment.

\section{Pitfalls of Experimenting in the Lab}

As every research method, lab experiments are not free from weaknesses, drawbacks, and risks. In this section we will point out potential pitfalls related to the lab experiment as a social process and introduce ways to address them. As in the following chapters on experiments in different settings, we will structure the discussion of potential pitfalls based on the three core elements of experimental designs: manipulation of treatment, group comparison, and random assignment.

\subsection{Caveats Concerning the Treatment}

The manipulation of a treatment variable is an essential part of every lab experiment. When analysing experimental data by comparing treatment and control group, researchers usually assume that subjects perceived the manipulated treatment as originally intended. However, this assumption doesn't necessarily hold in practice for several reasons. One problem can be that the treatment lacks sufficient salience or prominence. Sometimes treatments are just too weak to actually reach the subjects' awareness. Related to that, the experimental task itself or other aspects of the study might consume most of the subjects' attention and shift their cognitive focus away from the actual treatment. In both cases, the interpretation of a null effect of a

requirements. In sociology no clear standards have established, but the community currently goes more in the direction of economics with marked subfield specific variation. 
treatment becomes ambiguous, because the null effect can not only indicate that the treatment was actually ineffective but also that it wasn't sufficiently strong or salient in its current form in the chosen setting.

A further issue is that the treatment is too abstract or might convey a different meaning to the subjects than intended by the researchers. As a consequence, interpretation of results becomes misleading. For example, one common way to manipulate perceived ethnicity in experiments is to assign (hypothetical) names - such as typical "black" and "white" names - to interaction partners (see Lane 2016 for a meta-analysis on lab experiments on discrimination). However, these typical "black" and "white"" names are often confounded with subjects' perception of other - potentially relevant - traits, especially social economic status. Let's assume that the results of a experiment indicate that subjects cooperate less with a person with a typical "black" name than with another person with typical "white" name. Researchers might erroneously interpret this finding as a clear indication for the presence of discrimination against blacks, although it might be also driven by subject's concerns about lacking trustworthiness of low-income persons. As this example demonstrates the general finding whether the treatment influences the outcome remains uncontested in such cases, but the substantive interpretation and derived implications of the experimental results can totally change.

For these reasons, except for sufficiently salient and unambiguous treatments, it should be obligatory to conduct manipulation checks before, during, and/or after the experiment and to report their results in the publication (for a similar recommendation see Foschi 2014). The main aim of such manipulation checks is to test whether the treatment is salient, conveys the intended meaning, and, as assumed, causally influences mediating factors as theoretically assumed. Successful validation checks are thus essential to secure that experimental results are actually as internally valid as theoretically assumed. Manipulation checks can take different forms and can involve the collection of both quantitative and qualitative data. One typical type of manipulation check is to run a pilot of the experiment and survey participants after the experiment in a standardized questionnaire, in open interviews, or in group discussions about the experiment and its treatment. The same can also be done after completion of the actual experiment. However, without manipulation checks in pretests, this is risky since it might lead one to conclude that the treatment was ineffective and that all the efforts of data collection were useless. Another way to conduct manipulation checks is to measure variables that should or should not be directly influenced by the manipulation besides the outcome under investigation. For example, manipulating physical attractiveness of a person by means of a photograph one 
might not only ask subjects as how attractive but also as how healthy and youthful they perceive the depicted person - both traits known from previous research to correlate with physical attractiveness (Patzer 2007).

In addition, a huge literature shows that treatments can have very different effects depending upon the framing or labelling of a decision situation. For example, social psychologists have shown that labelling the very same task once as the "Wall Street Game" and once as the "Community Game" had substantial effects on subjects' willingness to cooperate with each other. In a study by Liberman et al. (2004) subjects played the prisoner's dilemma in which mutual cooperation with the other player is the collective desirable outcome but incentives for individual defection exist. Around 70 percent of the subjects nonetheless cooperated if the game was labelled as a "Community Game" whereas only about 30 percent were willing to cooperate in the very same game labelled as a "Wall Street Game". While framing effects are often not as strong as in this example and cannot be avoided completely, the lesson to take home from the presented study is that the presentation of the decision task deserves careful consideration, especially taking into account unwanted effects of labelling and framing.

Finally, the desire to implement sufficiently salient and strong treatments can researchers lead astray in the opposite direction when designing experiments: choosing unrealistically strong or too obvious treatments. In combination with the fact that subjects are aware of taking part in an experiment and being under observation, too strong or obvious treatments can limit generalizability of findings. Subjects might react differently to treatments in the lab than in the real world due to their knowledge of being observed by researchers (reactivity). This means that external validity in some lab experiments may be limited due to an interaction of the treatment effects with the particular design of the treatments and the lab setting. For example, as Keuschnigg and Wolbring (2015) highlight subjects might avoid discriminating minorities in the lab due to social desirability but might do so in the field when they feel unobserved. As we elaborate in more detail in the following subsection, reactivity - the fact that people behave differently if observed by a researcher - can be a major threat to the validity of inferences based on comparisons of treatment and control groups.

\subsection{Caveats Concerning Group Comparisons}

The basic idea of lab experiments is to let subjects complete a task and to intervene in the lab environment for a subset of all subjects. Subjects receiving and intervention function as treatment group, while the remaining subjects serve as a control. A simple comparison of mean 
outcomes between the treatment and the control group should inform about the causal effect of the treatment. In principle, this approach is straightforward and appears ideal for causal inference from a counterfactual perspective. However, besides the aforementioned spillover effects, other problems can undermine the validity of causal inferences based on such simple comparison of experimental groups.

Some of the most widely discussed issues in that context are so-called experimenter or demand effects. The problem arises due to interactions between experimental staff and subjects: The behaviour of experimental staff and the setup of the experiment can raise expectations about "adequate" behaviour in the experiment among the subjects and can lead them to systematically deviate from their "natural" reactions. Even worse, demand effects usually vary by treatment and are hence different for the treatment and the control group undermining group comparisons. As a consequence, demand effects often lead to experimental artefacts and can bias estimates in both directions: subjects may choose actions which they perceive to as "adequate" or expected in the specific situation (self-fulfilling prophecy) or they may try to do just the opposite and disconfirm expectations (self-destroying prophecy).

More specifically, the problem of such experimental artefacts can be divided into two broad categories: artefacts due to experimenter effects and due to subjects' expectations. A wellknown experiment highlighting the former - the potential influence of experimenters on experimental results - is the study by Rosenthal and Jacobson (1966). After conducting a general ability test in an elementary school in San Francisco, the researchers reported some students "to classroom teachers as showing unusual potential for intellectual gains" (p.115). Although it was in fact just randomly selected and hence unrelated to the actual test performance which students received this "high potential" label, IQ gains within 8 months after the intervention were much higher in the treated $(\Delta \mathrm{IQ}=12.2)$ than in the control group $(\Delta \mathrm{IQ}=8.4)$.

So why is this study about the effects of teacher expectations on kids' academic performance relevant for lab experiments? The equivalent to elementary school teachers being informed about some "high potential" students in the laboratory is the staff administering the treatment while knowing about the assignment of subjects to different treatment conditions. Even if the experimental staff is uninformed about the actual aim of the treatment and related research hypotheses, they will form expectations about the topic of the experiment and researchers' expectations of what to find. The experimental staff might thus - sometimes on purpose, but more frequently subconsciously - influence subjects' behaviour in the expected or the opposite 
direction by providing verbal and non-verbal cues which lead subjects in the treatment and/or control condition to behave differently (for a more detailed discussion see Bless et al. 1993).

A common way to circumvent such problems are so-called double-blind experiments, in which not only the subjects (single-blinding) but also the experimental staff (double blinding) is neither aware of the treatment nor which subjects received it. In theory, the rationale for double-blind experiments is straightforward: If nobody knows about the treatment, it is hard to form expectations and to adjust behaviour according to these expectations. However, in practice this is easier said than done, since administering the treatment usually requires some changes in the experimental setup, which might be easily recognized by the experimental staff. For example, in the treatment condition, the instructions might change, the sequence of the experimental stages might vary, or some additional task needs to be completed by the subjects. Experiments in medicine hence often rely on placebo treatments to make it harder for both subjects and staff to identify the treatment and assignment status of a subject. However, in social science experiments administering such placebos is rarely possible in practice.

A related but separate problem to artefacts due to experimenter effects are artefacts due to expectations on the subjects' side. On the one hand, this matter concerns reactivity - the fact that being aware of participating in a study leads subjects to change their behaviour in the treatment and/or control group. Clearly, this can undermine internal validity causing wrong inferences about causal effects. Besides this, lacking generalizability of findings beyond the lab may be another major consequence of reactivity, since subjects might have behaved differently in a more "natural" social environment. However, it appears worth emphasizing that whether and to what extent lab experimental findings can be generalized to other units, treatments, observations, and settings always depends - among others - on the particular lab experimental setup and the specific research question at hand. Claiming that lab experimental results always lack external validity is hence as hard to justify on scientific grounds as stating that external validity is never an issue for lab experiments.

On the other hand, subjects are not only aware that they participate in a scientific study, but - as staff members - they form expectations about potential treatments and the aim of the study. So-called "scientific do-gooders" (Ball and Cech 1996; see also Rosenthal and Rosnow 1973), which are overrepresented in lab experiments due to their high willingness of cooperate (see subsection 3.3), strive to meet the researchers expectations about attitudes and behaviour particularly well, while others will try to outsmart the researchers or to disprove the research hypothesis. The problem becomes especially pressing when subjects have stakes in the results 
of a study and thus try to influence the findings in a certain direction. Besides artefacts due to subjects' expectations, the overrepresentation of "scientific do-gooders" also highlights a more general point related to the random sampling and assignment of subjects discussed in more detail in the following subsection.

\subsection{Caveats Concerning Random Sampling and Assignment}

The optimal approach to recruit subjects to the lab would be random sampling from the population of interest. Since many lab experiments investigate human decision-making or human interactions, the exact population of interest is often rather general but seldom welldefined. Aiming to test general explanations about human behavior, it suggests itself to sample from the general population. However, as outlined above, it is rarely feasible in lab experiments without substantial effort to randomly sample from the population of interest. Therefore, lab experiments commonly rely on student subject pools, even if the actual target population is the general population or some group of professionals. Against this background, many discussions on lab experiments centre on their external validity regarding the units of observation. External validity would be limited, if the treatment effect interacts with the particular units under investigation. It is thereby an empirical question depending upon research topic and experimental design whether determinants of student behaviour in the lab are informative about other populations.

Furthermore, even if research using student samples focuses on this specific target population, the use of subject pools can create additional problems of selectivity. Students usually enrol in a subject pool on a voluntary basis or, in more technical terms, self-select into the subject pool. As explained in the previous section, these self-selected students have, on average, a higher willingness to cooperate, are more reliable, and show more interest in scientific research. In addition, students in a subject pool can have very specific motivations to participate in experiments including not only the aim to earn money, but also the entertainment of "playing" experimental games and a curiosity to learn more about experimental research. The latter particularly holds for students with a background in economics, social psychology, and other social and behavioural sciences. Depending upon the specific study programme, these students might behave differently than students with other majors or the general population. For example, several studies show that studying economics makes students behave more selfish and cooperate less with others (see Frank et al. 1993; Gerlach 2017). At the same time, for the above-mentioned reasons such as earning money, so-called "lab rats" (Guillen and Veszteg 
2012) frequently participate in experiments - sometimes even more than once in the same study, if researchers do not take precautions. Due to lab rats' familiarity with the lab situation and with specific experimental games (learning effect) as well as due to their often exceptional curiosity and/or monetary motivation (selection effect), these professional subjects might deviate from other participants in systematic and meaningful ways.

While one potential weakness of lab experiments concerns random sampling, a general strength of the lab setting is a high degree of control of the researcher over the assignment mechanisms. Consequently, random assignment usually works pretty well in lab experiments. One thing that can go wrong, however, regarding assignment is that groups of students with a specific background self-select into certain experimental sessions. Even though students are randomly assigned to experimental conditions, they have usually some degrees of freedom when choosing the exact time slots for the experimental session. Students in the same cohort and with the same major have similar schedules and, thus, have a higher likelihood to end up in the same experimental session. Such clustering of students is sometimes hard to avoid completely. However, since subjects usually interact with each other via computer networks and are not aware of the identity of the assigned interaction partners, such clustering is often much less problematic than it appears at first glance.

\section{Ethical and Practical Aspects}

Before running a lab experiment, researchers need to solve several practical questions, such as getting access to or setting up a subject pool, randomly selecting and assigning subjects, choosing the laboratory, recruiting and training the experimental staff, and acquiring funding to run the experiment. Since establishing a subject pool and motivating subjects to come to the lab can be time consuming and since funding for incentivizing decisions is not always available, it is common practice in some research areas such as social psychology to require students in university classes to participate in experiments to get hourly credit as a subject. While such an approach secures a sufficient number of observations, it raises the questions how valid decisions and responses of such participants are, since these students may be not very motivated to participate in the experiment and to show a lot of effort. In worst case, they may even try to undermine the experiment as some kind of "retaliation" against the "forced" participation. For these reasons, even though self-selected subject pools are also not free from problems (see subsection 3.3), we recommend to rely on them instead of forcing students to participate with potentially severe consequences for the validity of inferences. 
Another practical issue is determining the timing of outcome measurement. On the one hand, a sufficiently, but not too long time should have passed between treatment implementation and measurement of the outcome. Otherwise, the intervention might not have a fair chance to become effective (too early measurement) or its effect may have already faded (too late measurement). Getting the timing of measurement right can be therefore essential for finding or not finding a treatment effect in an experiment. Especially if it appears likely that the effect of a treatment change over time, we recommend to repeatedly measure the outcome variable to get a more fine-grained picture of the dynamics of the treatment effect over time. On the other hand, the available observation-window in lab experiments is quite limited making it hard to implement lasting treatments and to measure long-term effects of an intervention. Of course, it is, in principle, possible to follow-up on subjects or even invite them to another experimental session, but this usually requires quite some additional effort and often leads to substantial nonresponse and attrition. Thus, while possible in theory, follow-up measurement is rarely done in practice.

Finally, one important domain of discussion concerns ethical considerations about deception of potential and actual subjects. As stated above, if the experiment involves risks, subjects need to be informed about the potential downsides of participation in advance to make an informed choice. Social and behavioural scientists from the different disciplines unanimously agree on this point. It is thus standard practices to inform subjects about risks and to ask for approval by an IRB board before conducting lab experiments which raise such ethical concerns.

In contrast to this issue, much less agreement exists across different disciplines regarding the question to what extent subjects can be deceived about the actual experiment, about potential interaction partners, and about the consequences of their decisions (for an overview see Barrera and Simpson 2012). These disciplinary differences are not only important when publishing in journals of the respective field, but also when using existing laboratories set up by researchers from a specific field. For example, economists, sociologists, and psychologists disagree whether the extent of deception involved in Asch's experiment on group pressure is still acceptable. For example, in most economic labs a strict no deception rule exists implying that Asch-type experiments are strictly forbidden. Interestingly, one of the major arguments brought forward by economists against deception is not an ethical, but a practical one: Building up a reputation for deceptive experiments has unpredictable consequences for future experiments. Once a subject pool is "spoiled", subjects' expectation formation is hard to control increasing the risk of artefacts and invalid inferences. Psychological and sociological labs have 
usually less strict guidelines regarding deception. This reflects not only the long and successful tradition of psychological and sociological research in using such treatments, but also the analytical focus of these disciplines on variables that are sometimes hard to manipulate without deception. A strict no deception rule thus overly limits the space of experiments one can run in these fields, while the risks of deception are not perceived as severe as in economics. Besides these discipline-specific perspectives, in case of uncertainty about the adequacy of deception in a given experiment, we recommend to consult an IRB board to rule out ethical concerns.

\section{Five Lessons from this Chapter}

$\checkmark$ Laboratory experiments rely on the three design elements common to all experiments manipulation, group comparison, and randomization - and are staged in the rather abstract, sterile and controlled setting of a laboratory.

$\checkmark$ Conducting experiments in the laboratory provides not only the advantage of good replicability, but gives researchers strict control over setting, treatment manipulation, and measurement of independent and dependent variables.

$\checkmark$ Lab experiments create on an "idealized social system" and abstract from the complexities and dynamics of social reality to a certain degree.

$\checkmark$ Due to the artificial situation in the lab, manipulation checks are essential to secure treatment validity.

$\checkmark$ Demand effects and reactivity are major challenges in lab experiments and can undermine their internal validity. 


\section{References}

Asch, S. E. (1951): Effects of group pressure upon the modification and distortion of judgment. In H. Guetzkow (ed.), Groups, leadership and men. Pittsburgh, PA: Carnegie Press. Pp. 177-190.

Ball, S. B., \& Cech, P.-A. (1996): Subject pool choice and treatment effects in economic laboratory research. In R. M. Issac (ed.), Research in Experimental Economics. Greenwich: JAI Press. Pp. 239292.

Barrera, D. \& Simpson, B. (2012): Much ado about deception: Consequences of deceiving research participants in the social sciences. Sociological Methods \& Research 41: 383-413.

Bless, H. \& Burger, A.M. (2016): A closer look at social psychologists' silver bullet. Inevitable and evitable side effects of the experimental approach. Perspectives on Psychological Science 11: 296308.

Bless, H., Strack, F. \& Schwarz, N. (1993): The informative functions of research procedures: Bias and the logic of conversation. European Journal of Social Psychology 23: 149-165.

Bond, R., \& Smith, P.B. (1996): Culture and conformity: A meta-analysis of studies using Asch's (1952b, 1956) line judgment task. Psychological Bulletin 119: 111-137.

Falk, A. and Heckman, J.J. (2009): Lab experiments are a major source of knowledge in the social sciences. Science 326: 535-538.

Foschi, M. (2014): Hypotheses, operationalizations, and manipulation checks. In M. Webster, Jr. \& J. Sell (eds.), Laboratory Experiments in the Social Sciences (2 ${ }^{\text {nd }}$ edition). San Diego, CA: Elsevier. Pp. 247-268.

Frank, R.H., Gilovich, T., \& Regan, D.T. (1993): Does studying economics inhibit cooperation? Journal of Economic Perspectives 7: 159-171.

Fréchette, G.R. (2015): Laboratory experiments: Professionals versus students. In G.R. Fréchette \& A. Schotter (eds.), Handbook of Experimental Economic Methodology. Oxford University Press. Pp360-390.

Gerlach, P. (2017): The games economists play: Why economics students behave more selfishly than other students. PLoS ONE 12: e0183814.

Guillen, P, \& Veszteg, R. (2012): On "lab rats". Journal of Socio-Economics 41: 714-720.

Güth, W., Schmittberger, R. \& Schwarze, B. (1982): An experimental analysis of ultimatum bargaining. Journal of Economic Behavior \& Organization 3: 367-388.

Hamermesh, D.S. \& Parker, A.M. (2005): Beauty in the classroom. Instructors' pulchritude and putative pedagogical productivity. Economics of Education Review 24: 369-376.

Keuschnigg, M., \& Wolbring, T. (2016): The use of field experiments to study mechanisms of discrimination. Analyse \& Kritik 38: 179-201.

Lane, T. (2016): Discrimination in the laboratory: A meta-analysis of economics experiments. European Economic Review 90: 375-402.

Liberman, V., Samuels, S. \& Ross, L. (2004): The name of the game: Predictive power of reputation vs. situational labels in determining prisoner's dilemma game moves. Personality and Social Psychology Bulletin 30: 1175-1185.

McLeod, S.A. (2008). Asch experiment. Retrieved from https://www.simplypsychology.org/aschconformity.html

Patzer, G.L. (2007):Why Physically Attractive People Are More Successful. The Scientific Explanation, Social Consequences, and Ethical Problems. New York: Edwin Mellen Press.

Rosenthal, R.W. \& Jacobson, L. (1966): Teachers expectancies: Determinants of pupils IQ gains. Psychological Reports 19: 115-118.

Rosenthal, R.W. \& Rosnow, R.L. (1973): The Volunteer Subject. New York: Wiley.

Shelly, R.K. (2007): Training Interviewers and Experimenters. In M. Webster, Jr. \& J. Sell (eds.), Laboratory Experiments in the Social Sciences (2 ${ }^{\text {nd }}$ edition). San Diego, CA: Elsevier. Pp. 83-102. 
Willer, David \& Walker, H.A. (2007): Building Experiments. Testing Social Theory. Stanford: Stanford University Press.

Wolbring, T. \& Riordan, P. (2016): How beauty works. Theoretical mechanisms and two empirical applications on students' evaluation of teaching. Social Science Research 57: 253-272. 